\title{
The mucin phenotype does not affect the endoscopic resection outcome of non-ampullary duodenal epithelial tumors
}

\section{다)(우우}

\author{
Authors \\ Institutions \\ 1 Division of Gastroenterology, Department of Internal \\ Medicine, School of Medicine, Iwate Medical University, \\ Yahaba, Japan \\ 2 Kaiunbashi Endoscopy Clinic, Morioka, Japan \\ 3 Division of Molecular Diagnostic Pathology, Department \\ of Pathology, School of Medicine, Iwate Medical \\ University, Yahaba, Japan
}

Yosuke Toya', Masaki Endoํ, ${ }^{1,}$, Shun Yamada ${ }^{1}$, Tomofumi Oizumi ${ }^{1}$, Toshifumi Morishita ${ }^{1}$, Risaburo Akasaka ${ }^{1}$, Shunichi Yanai $^{1}$, Shotaro Nakamura ${ }^{1}$, Makoto Eizuka ${ }^{3}$, Noriyuki Uesugi ${ }^{3}$, Tamotsu Sugai ${ }^{3}$, Takayuki Matsumoto ${ }^{1}$

submitted 22.12.2020

accepted after revision 8.3.2021

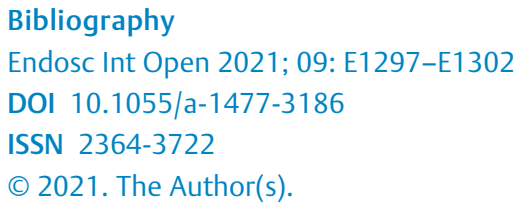
Commons Attribution-NonDerivative-NonCommercial License, permitting copying and reproduction so long as the original work is given appropriate credit. Contents may not be used for commercial purposes, or adapted, remixed, transformed or built upon. (https://creativecommons.org/licenses/by-nc-nd/4.0/)

Georg Thieme Verlag KG, Rüdigerstraße 14,

70469 Stuttgart, Germany

Corresponding author

Yosuke Toya, MD, PhD, Division of Gastroenterology, Department of Internal Medicine, Iwate Medical University School of Medicine, Idaidori 1-1-1, Yahaba 028-3694, Japan Fax: +81-19-907-7166

ytoya@iwate-med.ac.jp

\section{ABSTRACT}

Background and study aims Some studies have reported an association between clinicopathological features and mucin phenotypes of non-ampullary duodenal epithelial tumors (NADETs). However, the association between clinical outcomes of endoscopic resection (ER) and mucin phenotypes has not been elucidated. The aim of this retrospective study was to analyze clinical outcomes of ER of NADETs with reference to mucin phenotypes.

Patients and methods We retrospectively evaluated the clinical outcomes of ER for NADETs performed from 2006 to 2019 and compared clinicopathological characteristics, ER procedures, and outcomes, including adverse events (AEs) among tumors classified by mucin phenotype. Mucin phenotypes were classified as gastric, gastrointestinal, and intestinal based on immunohistochemical examination. Grade of dysplasia was determined according to the Vienna classification (VCL).

Results The proportion of VCL 4/5 was higher in the gastric type (50\%) compared with that in the gastrointestinal (39.1\%, $P=0.009$ ) and intestinal types (5.4\%, $P=0.008)$, respectively. With no statistical difference in tumor size and ER method among the three groups, no significant difference was observed for ER outcomes, i.e., en bloc and R0 resection rates. In the gastrointestinal and intestinal types, AEs occurred in four cases treated with ESD, but none developed in the gastric type.

Conclusions This study suggests that the mucin phenotype does not affect resection outcome. However, considering high malignant potential and tendency for low $A E$ rates, the gastric type NADETs may be more appropriate for proactive ER than the others.

\section{Introduction}

It is well known that endoscopic resection (ER) for non-ampullary duodenal epithelial tumors (NADETs) is difficult due to anatomical reasons [1-4]. Recently, the efficacy of some ER methods, such as cold polypectomy [5] and underwater endoscopic mucosal resection (UEMR) $[6,7]$, has been reported. In addi- tion, methods for prevention of delayed adverse events (AEs) after ER, such as line-assisted closure [8], endoscopic nasobiliary and pancreatic duct drainage (ENBPD) [9], and over-thescope clip closure $[10,11]$, have also been applied in several institutions. However, the strategy of ER for NADETs has not been fully standardized to date. 
Some studies have focused on the association between clinicopathological features and immunohistochemical mucin phenotypes of NADETs [12-18]. Those reports have shown that NADETs of the gastric phenotype have a higher malignant potential than those of the intestinal type. Recently, several groups of investigators reported on long-term outcomes of ER of NADETs [19-22]. However, the association between clinical outcomes of ER and mucin phenotypes has not been elucidated in detail. The aim of this study was to determine the practical efficacy and safety of ER for NADETs with special reference to the mucin phenotype of the tumor.

\section{Patients and methods}

\section{Patients}

Among 134 patients diagnosed with NADETs at Iwate Medical University Hospital from February 2006 to September 2019, we excluded patients with familial adenomatous polyposis, those who were not treated by ER, and those who had prior history of ER for NADETs. Consequently, a total of 70 lesions found in 70 patients treated by ER were enrolled in the present study. The medical charts of the patients were reviewed to collect demographic and clinical outcomes. Written informed consent was obtained from all patients before ER. Informed consent for study enrollment was obtained in the form of an opt-out button on the website. The study protocol was approved by the institutional review board of Iwate Medical University (No. MH2019154).

\section{Histological and immunohistochemical examinations}

All of the lesions were graded histologically according to the Vienna classification [23] (VCL) as either category 3 (C3, lowgrade adenoma/dysplasia) or category 4/5 (C4/5, mucosal high-grade neoplasia/submucosal invasion by carcinoma).

Immunohistochemical examination was performed with an auto-immunostaining system (Dako EnVision System, Denmark). On the basis of our previous report [14,24], we performed immunohistochemical staining using antibodies for MUC2 (Ccp58; Novocastra Laboratories, Newcastle, UK, dilution 1:100), MUC5AC (CLH2; Novocastra Laboratories, Newcastle, UK, dilution 1:100), MUC6 (CLH5; Novocastra Laboratories Newcastle, UK, dilution 1:100), and CD10 (56C56; Novocastra Laboratories, dilution $1: 80$ ) to determine tumor histological phenotype. The gastric type was defined as a phenotype positive for only gastric markers (MUC5AC and MUC6). The intestinal type was defined as a phenotype positive for only intestinal markers (MUC2 and CD10). Tumors positive for both gastric and intestinal phenotypes were regarded as having a gastrointestinal phenotype [25]. We defined the percentage of tumor cells with positive expression $>10 \%$ as positive expression of the markers in the lesions [26].

\section{Endoscopic resection of NADETs}

The choice of either endoscopic mucosal resection (EMR) or endoscopic submucosal dissection (ESD) was decided mainly based on lesion size. EMR was selected for NADETs $<20 \mathrm{~mm}$.
ESD was selected for tumors $>20 \mathrm{~mm}$ or in cases in which severe fibrosis is expected from previous biopsy before treatment. EMR and ESD were performed principally with a therapeutic endoscope (GIF-Q260 J, Olympus, Japan). We used double-balIoon endoscopy (EC450-BI5, Fujifilm Corp, Japan) for some lesions located distal to the papilla of Vater. EMR procedures consisted of submucosal injection followed by mucosal resection with a snare (Captivator II, Boston Scientific, United States). Either a hook knife (KD-620QR, Olympus, Japan) or a combination of a hook knife and the SB Knife Jr (Sumitomo Bakelite, Japan) was applied to the ESD procedure. Post-ER ulcers were closed principally by simple closure with clips to prevent delayed bleeding or perforation. The ulcer in a case of EMR was closed with the over-the scope clip (Ovesco Endoscopy, Germany). If the ulcer was difficult to close completely, laparoscopic closure was performed.

Intraoperative perforation was defined as a defect in the muscle layer that was confirmed during the ER procedure. We performed computed tomography (CT) only for cases suspected of perforation during or after ER. Retroperitoneal emphysema was defined as free air in only the retroperitoneum, as detected by $\mathrm{CT}$ without peritoneal irritation symptoms and abscess formation. Local recurrence was defined as endoscopic or pathological confirmed recurrence after ER at the prior resection site.

\section{Statistical analyses}

Non-categorical variables are indicated as median, range, and interquartile range (IQR). Categorical variables are indicated as frequency and percent. The clinicopathological and ER outcomes of the subjects were compared among the three mucin phenotypes with the Kruskal-Wallis test. When there was a statistically significant difference among the three groups, any two groups were compared with a chi-square test or Fisher's exact probability test. For multiple comparisons, probability was adjusted by Bonferroni correction. $P<0.05$ was considered to be statistically significant. All of the statistical analyses were performed with SPSS version 25 software for MAC OS (SPSS Inc., Chicago, Illinois, United States).

\section{Results}

- Table 1 shows the clinicopathological characteristics of the study subjects. The median age was 67 years, with a predominance of males (65.7\%). The most frequent location was the second portion, and the median tumor size was $10 \mathrm{~mm}$. Fiftythree lesions (75.7\%) were the elevated type. Forty-four lesions (62.9\%) were treated by EMR, and 26 lesions (37.1\%) were treated by ESD. The median procedure duration of EMR and ESD was 6 minutes and 56 minutes, respectively. Conventional clips were used as the closing method in most cases. Only one case was closed with an over-the scope clip, and two cases required laparoscopic closure because of incomplete closure by conventional clips or intraoperative perforation.

Immunohistochemical examinations revealed that 10 lesions (14.3\%) were the gastric type, 23 lesions (32.9\%) were the gastrointestinal type, and 37 lesions (52.9\%) were the 
- Table 1 Clinicopathological characteristics of 70 patients with NADETs

\begin{tabular}{|c|c|}
\hline \multicolumn{2}{|l|}{ Variables $(n=70)$} \\
\hline Age, years, median (range, IQR) & $67(44-82,15)$ \\
\hline \multicolumn{2}{|l|}{ Sex, n (\%) } \\
\hline - Male & $46(65.7)$ \\
\hline - Female & $24(34.3)$ \\
\hline \multicolumn{2}{|l|}{ Tumor location, n (\%) } \\
\hline - Bulbs & $19(27.1)$ \\
\hline - Second portion & $48(68.6)$ \\
\hline - Third portion & $3(4.3)$ \\
\hline - Tumor size, mm, median (range, IQR) & $10(2-47,9)$ \\
\hline \multicolumn{2}{|l|}{ Macroscopic appearance, n (\%) } \\
\hline - Elevated type & $53(75.7)$ \\
\hline - Depressed type & $17(24.3)$ \\
\hline \multicolumn{2}{|l|}{ Therapeutic method, n (\%) } \\
\hline - EMR & $44(62.9)$ \\
\hline - ESD & $26(37.1)$ \\
\hline \multicolumn{2}{|l|}{ Procedure duration, min, median (range, IQR) } \\
\hline - EMR & $6(2-24,8)$ \\
\hline . ESD & $56(7-270,99)$ \\
\hline \multicolumn{2}{|l|}{ Closing method, n (\%) } \\
\hline - Conventional clip & $67(95.7)$ \\
\hline - Over-the-scope clip & $1(1.4)$ \\
\hline - Laparoscopic closure & $2(2.9)$ \\
\hline \multicolumn{2}{|l|}{ Mucin phenotype, n (\%) } \\
\hline - Gastric type & $10(14.3)$ \\
\hline - Gastrointestinal type & $23(32.9)$ \\
\hline - Intestinal type & $37(52.9)$ \\
\hline \multicolumn{2}{|l|}{ VCL, n (\%) } \\
\hline - Category 3 & $54(77.1)$ \\
\hline - Category 4/5 & $16(22.9)$ \\
\hline $\begin{array}{l}\text { Follow-up period, months, median (range, } \\
\text { IQR) }\end{array}$ & $39.5(2-170,100)$ \\
\hline
\end{tabular}

Local recurrence, $\mathrm{n}(\%)$

$2(2.9)$

NADET, non-ampullary duodenal epithelial tumor; IQR, interquartile range; EMR, endoscopic mucosal resection; ESD, endoscopic submucosal dissection; VCL, Vienna classification.

intestinal type. According to VCL, 54 lesions (77.1\%) were regarded as category 3, and 16 lesions (22.9\%) as category 4 or 5. The median follow-up period was 39.5 months, and local recurrence was observed in two lesions (2.9\%).
- Table 2 shows the comparison of clinicopathological and ER outcomes among the three groups classified by mucin phenotypes. Tumor location was significantly different among the three mucin phenotypes. Lesions of the gastric type were more frequently located in the bulbs than those of the gastrointestinal type $(P<0.001)$ and the intestinal type $(P<0.001)$. Tumor size was not significantly different among the three mucin phenotypes. The proportion of VCL 4/5 was significantly different between the gastric and the intestinal types $(P=0.009)$ and between the gastrointestinal and the intestinal types $(P=$ $0.008)$. Choice of ER method was not significantly different among the three groups. No significant differences were observed among the three mucin phenotypes with respect to en bloc resection and $\mathrm{R} 0$ resection. The rate of $A E s$ with $E R$ was not significantly different among the three mucin phenotypes. However, there were no AEs with ER for lesions of the gastric phenotype.

Clinicopathological characteristics of four cases with AEs are summarized in $>$ Table 3 . All cases were treated by ESD, and except for Case 1, tumor size was $>20 \mathrm{~mm}$, with procedure duration over 180 minutes. The mucin phenotypes of the cases were either the gastrointestinal or the intestinal types. Only a single case was classified as VCL category 4. Intraoperative perforation and retroperitoneal emphysema were each observed in two cases. Two of four cases required laparoscopic closure due to incomplete closure for intraoperative perforation by conventional clip. The remaining two cases were managed conservatively after endoscopic closure with Endoclip.

\section{Discussion}

In the present investigation, we have shown an association between the clinical outcomes of ER and the mucin phenotype of NADETs. According to the mucin phenotypes, location in bulbs and VCL 4/5 were significantly more common with the gastric phenotype. Finally, we found that no AEs occurred with ER for lesions of the gastric phenotype. To our knowledge, this is the first study to examine the association between clinical outcomes of ER and mucin phenotypes of NADETs.

Our data showed that AEs occurred only during the ESD procedure. Recently, several studies have shown the clinical outcomes of ER of NADETs [19-21,27]. These reports showed that EMR is more suitable and safer than ESD, especially for small lesions. On the other hand, ESD can achieve complete resection, even for large lesions. However, several studies have reported that ESD is associated with more AEs, such as bleeding and perforation $[1,2,27,28]$. Considering the much higher risk of AEs with ESD, several endoscopic methods, such as line-assisted closure [8], ENBPD [9] and over-the-scope clip closure $[10,11]$ have been introduced for the prevention of AEs. More recently, Kanaji et al reported the safety and feasibility of laparoscopic cooperative surgery for duodenal tumors (D-LECS) [29]. In the present study, AEs occurred in four cases treated by ESD. However, all of those cases were treated conservatively after conventional clip closure or laparoscopic closure without additional surgery, such as pancreatoduodenectomy. Further evaluation in a prospective multicenter study is needed to de- 
- Table 2 Comparison of clinicopathological characteristics and ER outcomes of three mucin phenotypes

\begin{tabular}{|c|c|c|c|c|}
\hline & Gastric type $(n=10)$ & Gastrointestinal type $(n=23)$ & Intestinal type $(n=37)$ & $P$ value \\
\hline \multicolumn{5}{|l|}{ Tumor location, n (\%) } \\
\hline - Bulbs & $10(100)$ & $6(26.1)$ & $3(8.1)$ & $<0.001^{1}$ \\
\hline - Second portion & $0(0)$ & $17(73.9)$ & $31(83.8)$ & \\
\hline - Third portion & $0(0)$ & $0(0)$ & $3(8.1)$ & \\
\hline Tumor size, mm, median (range, IQR) & $12(3-30,12)$ & $11(4-40,8)$ & $8(2-47,11)$ & 0.076 \\
\hline \multicolumn{5}{|l|}{ VCL, n (\%) } \\
\hline - Category 4/5 & $5(50)$ & $9(39.1)$ & $2(5.4)$ & $0.001^{2}$ \\
\hline \multicolumn{5}{|l|}{ Therapeutic method, n (\%) } \\
\hline - EMR & $4(40)$ & $13(56.5)$ & $27(73)$ & 0.15 \\
\hline - ESD & $6(60)$ & $10(43.5)$ & $10(27)$ & \\
\hline En bloc resection, n (\%) & $10(100)$ & $20(87.0)$ & $34(91.9)$ & 0.47 \\
\hline R0 resection, n (\%) & $90(90)$ & $18(78.3)$ & $24(64.9)$ & 0.23 \\
\hline Intraoperative perforation, n (\%) & $0(0)$ & $1(4.3)$ & $1(2.7)$ & 0.79 \\
\hline Retroperitoneal emphysema, n (\%) & $0(0)$ & $1(4.3)$ & $1(2.7)$ & 0.79 \\
\hline
\end{tabular}

- Table 3 Clinicopathological characteristics of four cases with an adverse events

\begin{tabular}{|l|l|l|l|l|}
\hline & Case 1 & Case 2 & Case 3 & Case 4 \\
\hline Therapeutic method & ESD & ESD & ESD & ESD \\
\hline Tumor size, $\mathrm{mm}$ & 4 & 24 & 33 & 40 \\
\hline Tumor location & Second portion & Second portion & Third portion & Second portion \\
\hline Procedure time, min & 76 & 189 & 270 & 246 \\
\hline Mucin phenotype & Intestinal & Gastrointestinal & Intestinal & Gastrointestinal \\
\hline VCL & Category 3 & Category 4 & Category 3 & Category 3 \\
\hline Adverse event & Intraoperative perforation & Intraoperative perforation & Retroperitoneal emphysema & Retroperitoneal emphysema \\
\hline Closing method & Conventional clip & Laparoscopic closure & Conventional clip & Laparoscopic closure \\
\hline ESD, endoscopic submucosal dissection; VCL, Vienna classification. & &
\end{tabular}

termine suitable methods for the prevention of AEs after ER of NADETs, especially ESD.

Our data showed that there were no AEs, such as perforation or bleeding, with the EMR procedure. However, conventional EMR was reported to be accompanied by AEs, such as bleeding ( $0 \%$ to $12 \%$ ) and perforation ( $0 \%$ to $5 \%$ ) $[1-3,19-21,22,27]$. Accordingly, new methods, such as cold polypectomy and underwater EMR, have been introduced recently. Maruoka et al reported that there was no delayed bleeding or intraprocedural/ delayed perforation after cold polypectomy for small, sporadic, non-ampullary duodenal adenomas [5]. Yamasaki et al showed that underwater EMR achieved a high complete resection rate without AEs [6]. These modified ER procedures may be more frequently applied in the future because of their safety and convenience.

We have previously reported on correlations of the mucin phenotypes of NADETs with tumor location, endoscopic findings including magnifying endoscopy, and malignant potential $[14,24]$. Our data showed that the proportion of VCL 4/5 was significantly higher in the gastric type than in the intestinal type. We also reported previously that it was possible to predict the mucin phenotype of NADETs by the site and the conventional and magnifying endoscopic findings [14]. In the present study, we could show that there were no AEs with ER for lesions of the gastric phenotype. That may be a consequence of the location of the lesions, because lesions of the gastric type were 
located predominantly in the duodenal bulbs. These results may be explained by the fact that the bulbar location is less prone to bile and pancreatic juice, thereby resulting in the safety of ER. It thus seems possible that mucin phenotype of NADETs, together with the location, may be a hallmark for the prediction of AEs after ER.

In the present study, the proportion of VCL 4/5 was significantly higher in the gastric and gastrointestinal types than in the intestinal type. However, we failed to show any clear differences in the clinicopathological characteristics and ER outcomes between gastric and gastrointestinal phenotypes. Further investigations with a larger number of cases are warranted elucidate this issue.

The present study has several limitations. First, the retrospective nature of the study at a single center introduced selection bias. However, we believe our data to be representative of the practical clinical situation in the management of NADETs. Second, the sample size was too small to proceed with statistical analysis of the AEs of ER and for NADETs. A multicenter, prospective study is warranted to validate our findings.

\section{Conclusions}

In conclusion, our study confirmed that ER outcomes such as en bloc resection rate, $\mathrm{R} 0$ resection rate, and tumor size were not significantly different among the three mucin phenotypes. However, the AEs did not occur in the lesions with gastric phenotype. These findings suggest that the mucin phenotype should not necessarily be included in the selection criteria for ER in patients with NADETs. However, the gastric phenotype may be appropriate candidates for ER in consideration of their high malignant potential and low risk of AEs.

\section{Competing interests}

The authors declare that they have no conflict of interest.

\section{References}

[1] Endo M, Abiko Y, Oana S et al. Usefulness of endoscopic treatment for duodenal adenoma. Dig Endosc 2010; 22: 360-365

[2] Nonaka S, Oda I, Tada K et al. Clinical outcome of endoscopic resection for nonampullary duodenal tumors. Endoscopy 2015; 47: 129135

[3] Inoue T, Uedo N, Yamashina T et al. Delayed perforation: a hazardous complication of endoscopic resection for non-ampullary duodenal neoplasm. Dig Endosc 2014; 26: 220-227

[4] Ochiai Y, Kato M, Kiguchi Y et al. Current status and challenges of endoscopic treatment for duodenal tumors. Digestion 2019; 99: 2126

[5] Maruoka D, Matsumura T, Kasamatsu S et al. Cold polypectomy for duodenal adenomas: a prospective clinical trial. Endoscopy 2017; 49: 776-783

[6] Yamasaki Y, Uedo N, Takeuchi Y et al. Underwater endoscopic mucosal resection for superficial nonampullary duodenal adenomas. Endoscopy 2018; 50: 154-158
[7] Iwagami H, Takeuchi Y, Yamasaki Y et al. Feasibility of underwater endoscopic mucosal resection and management of residues for superficial non-ampullary duodenal epithelial neoplasms. Dig Endosc 2020; 32: 565-573

[8] Yamasaki Y, Takeuchi Y, Uedo N et al. Line-assisted complete closure of duodenal mucosal defects after underwater endoscopic mucosal resection. Endoscopy 2017; 49: E37-E38

[9] Fukuhara S, Kato M, Iwasaki E et al. Management of perforation related to endoscopic submucosal dissection for superficial duodenal epithelial tumors. Gastrointest Endosc 2020; 91: 1129-1137

[10] Tashima T, Ohata K, Sakai E et al. Efficacy of an over-the-scope clip to overcome the problem. Scope clip for preventing adverse events after duodenal endoscopic submucosal dissection: a prospective interventional study. Endoscopy 2018; 50: 487-496

[11] Dohi O, Yoshida N, Naito Y et al. Efficacy and safety of endoscopic submucosal dissection using a scissors-type knife with prophylactic over-the-scope clip closure for superficial non-ampullary duodenal epithelial tumors. Dig Endosc 2020; 32: 904-913

[12] Toba T, Inoshita N, Kaise M et al. Clinicopathological features of superficial non-ampullary duodenal epithelial tumor; gastric phenotype of histology correlates to higher malignant potency. J Gastroenterol 2018; 53: 64-70

[13] Mitsuishi T, Hamatani S, Hirooka S et al. Clinicopathological characteristics of duodenal epithelial neoplasms: Focus on tumors with a gastric mucin phenotype (pyloric gland-type tumors). PLoS One 2017; 12: e0174985

[14] Toya Y, Endo M, Akasaka R et al. Clinicopathological features and magnifying chromoendoscopic findings of non-ampullary duodenal epithelial tumors. Digestion 2018; 97: 219-227

[15] Minatsuki C, Yamamichi N, Inada K et al. Expression of gastric markers is associated with malignant potential of nonampillary duodenal adenocarcinoma. Dig Dis Sci 2018; 63: 2617-2625

[16] Yoshida M, Shimoda T, Abe M et al. Clinicopathological characteristics of non-ampullary duodenal tumors and their phenotypic classification. Pathol Int 2019; 69: 398-406

[17] Akazawa Y, Ueyama H, Tsuyama S et al. Endoscopic and clinicopathological features of superficial non-ampullary duodenal tumor based on the mucin phenotypes. Digestion 2020: 1-8. doi:10.1159/ 000508040. Online ahead of print.

[18] Matsueda K, Uedo N, Kitamura M et al. Pre-ampullary location and size $\geq 10 \mathrm{~mm}$ are independent predictors for high-grade superficial non-ampullary duodenal epithelial tumors. J Gastroenterol Hepatol 2020: doi:10.1111/jgh.15317

[19] Tomizawa Y, Ginsberg GG. Clinical outcome of EMR of sporadic, nonampullary, duodenal adenomas: a 10-year retrospective. Gastrointest Endosc 2018; 87: 1270-1278

[20] Valerii G, Tringali A, Landi R et al. Endoscopic mucosal resection of non-ampullary sporadic duodenal adenomas: a retrospective analysis with long-term follow up. Scand J Gastroenterol 2018; 53: 490-494

[21] Hara Y, Goda K, Dobashi A et al. Short- and long-term outcomes of endoscopically treated superficial non-ampullary duodenal epithelial tumors. World J Gastroenterol 2019; 25: 707-718

[22] Hwang KL, Kim GH, Lee BE et al. Long-term outcomes of endoscopic resection for non-ampullary duodenal epithelial tumors: A singlecenter experience. Turk J Gastroenterol 2020; 1: 49-57

[23] Dixon MF. Gastrointestinal epithelial neoplasia: Vienna revised. Gut 2002; 51: 130-131

[24] Toya Y, Endo M, Oizumi T et al. Diagnostic algorithm of magnifying endoscopy with crystal violet staining for non-ampullary duodenal epithelial tumors. Dig Endosc 2020; 32: 1066-1073

[25] Kobayashi M, Takeuchi M, Ajioka Y et al. Mucin phenotype and narrow-band imaging with magnifying endoscopy for differentiatedtype mucosal gastric cancer. J Gastroenterol 2011; 46: 1064-1070 
[26] Yao T, Takata M, Tustsumi S et al. Phenotypic expression of gastrointestinal differentiation markers in colorectal adenocarcinomas with liver metastasis. Pathology 2002; 34: 556-560

[27] Yahagi N, Kato M, Ochiai Y et al. Outcomes of endoscopic resection for superficial duodenal epithelial neoplasia. Gastrointest Endosc 2018; 88: 676-682
[28] Hoteya S, Furuhata T, Takahito T et al. Endoscopic submucosal dissection and endoscopic mucosal resection for non-ampullary superficial duodenal tumor. Digestion 2017; 95: 36-42

[29] Kanaji S, Morita Y, Yamazaki Y et al. Feasibility of laparoscopic endoscopic cooperative surgery for non-ampullary superficial duodenal neoplasms: single-arm confirmatory trial. Dig Endosc 2020; 33: 373380 